\title{
Simultaneous Cyclic Scheduling and Control of Tubular Reactors: Single Production Lines
}

\author{
Antonio Flores-Tlacuahuac* \\ Departamento de Ingeniería y Ciencias Químicas, Universidad Iberoamericana \\ Prolongación Paseo de la Reforma 880, México D.F., 01210, México \\ Ignacio E. Grossmann \\ Department of Chemical Engineering, Carnegie-Mellon University \\ 5000 Forbes Av., Pittsburgh 15213, PA
}

July 19, 2010

*Author to whom correspondence should be addressed. E-mail: antonio.flores@uia.mx, phone/fax: +52(55)59504074, http://200.13.98.241/ antonio 


\begin{abstract}
In this work we propose a simultaneous scheduling and control optimization formulation to address both optimal steady-state production and dynamic product transitions in continuous multiproduct tubular reactors. The simultaneous scheduling and control problem for continuous multiproduct tubular reactors is cast as a Mixed-Integer Dynamic Optimization (MIDO) problem. The dynamic behavior of the tubular reactor is represented by a set of nonlinear partial differential equations that are merged with the set of algebraic equations representing the optimal schedule production model. By using the method of lines, the process dynamic behavior is approximated by a set of nonlinear ordinary differential equations. Moreover, time discretization of the underlying system allows us to transform the problem into a Mixed-Integer Nonlinear Programming (MINLP) problem. Three multiproduct continuous tubular reactors are used as examples for testing the simultaneous scheduling and control optimization formulation.
\end{abstract}




\section{Introduction}

Modern society demands large amounts of some commodities such as polymer products. In fact, most of these products, such as polyethylene, poly-methyl-methacrylate, polystyrene, etc., are manufactured using continuous production facilities, rather than batch or semi-batch equipment. The reason is that continuous production plants are able to significantly increase productivity in comparison with noncontinuous processes. In the specific case of the polymer industry continuous stirred tank reactors (CSTR), tubular reactors (PFR), or a variation/combination of them, have been used as continuous plants for manufacturing polymers. Each one of them presents advantages and disadvantages. For instance, it is widely known that polymerization reactors carried out in autoclaves are prone to suffer runaway temperature problems because of the presence of hot spots [1]. Moreover, because some autoclave polymerization reactors are operated around extreme temperature and pressure processing conditions, acceptable closed-loop control can be hard to achieve. Operability problems faced by CSTRs are instead usually better handled by using tubular reactors. In fact, better control temperature in highly exothermic polymerization reaction systems can be achieved by carrying out such reactions in PFRs facilities. Moreover, a single PFR can feature the same productivity targets as a battery of CSTRs, potentially leading to a reduction in capital and operating costs. Mathematical modeling, in conjunction with a strong experimental program is a powerful way to improve our understanding of complex reaction processes. However, it should be stressed that both dynamic simulation and optimization of PFRs are harder to perform because of the distributed nature of tubular reactors. Moreover, the highly nonlinear nature of many industrially relevant reactions in terms of multiple steady-states and oscillatory behavior, to name just a few nonlinearity patterns, leads to difficult mathematical models especially for process optimization purposes.

The common practice in scheduling and control problems has been to solve them independently. The solution of scheduling problems provides information regarding the best production sequence, amounts to be produced, production times, etc, given a profitability index that is to be optimized. In this context, process dynamics is neglected leading, for instance, to assume constant transition times among all products to be manufactured. On the other hand, the solution of control problems renders optimal values of the selected manipulated variables leading, for instance, to minimum transition times. Normally, during 
transition operations, minimum off-spec material should be also an operation target. During the solution of control problems we normally assume that the production sequence has been fixed. This assumption allows us to solve optimal control problems independently of the underlying scheduling problem. However, it has been widely recognized [2, [3],[4],[5], that scheduling and control problems are tightly integrated problems and that improved optimal solutions can be obtained by solving both problems simultaneously rather than independently, because interactions between problems are explicitly taken into account [6], [7]. A literature review on scheduling and control problems can be found elsewhere [6].

In this work we extend the results reported in [6] to address the simultaneous scheduling and control problem in a single tubular reactor that manufactures multiple products. As was done in [6], we merge optimization formulations for dealing with scheduling and optimal control problems. Working along these lines, the problem is cast as an optimization problem featuring integer and continuous variables, and because states and manipulated variables are time dependent, the underlying optimization problem is a mixed-integer dynamic optimization (MIDO) problem. To solve the MIDO problem we have selected to transform it into a mixed-inter nonlinear program (MINLP). We should stress that because tubular reactors represent distributed parameter systems, its numerical treatment is normally harder to deal with than for aggregated systems (the type of dynamic systems addressed in [6]). This is especially true for the optimization of such a distributed parameter systems. The proposed strategy for solving MIDO problems featuring distributed parameter systems consists of the spatial and time discretization of the PDAE system leading to a final set of nonlinear equations. The spatial discretization is approached by using the method of lines [8], whereas the temporal discretization is addressed using the orthogonal collocation method on finite elements [9]. Because the resulting MINLP formulation features non-convexities, the solution of the underlying MINLP was obtained by using the sbb/CONOPT solver embedded in the gams environment. Recently some open source software for MINLP has become available [10]. Moreover, we propose the use of a decomposition method for addressing the solution of the scheduling and control problems of the MIDO case studies addressed in this work. This point is relevant because without an efficient initialization and decomposition method most of the large scale MIDO problems (as the ones addressed in the present work) turns out to be difficult or impossible to solve. 


\section{Problem definition}

Given are a number of products that are to be manufactured in a single continuous multiproduct PFR. The corresponding steady-state operating conditions for the production of each product are computed as part of the solution strategy. The demand rate, the cost of products, raw materials and inventories must be also specified. Using this information and the SC optimization formulation the problem consists of the simultaneous determination of the best production wheel (i.e. cyclic time and the sequence in which the products will be manufactured) as well as the transition times, production rates, length of processing times, amounts manufactured of each product, such that the profit is maximized subject to a set of scheduling and dynamic state constraints.

\section{Scheduling and Control MIDO Formulation}

To tackle the simultaneous scheduling and control (SSC) problem, we assume that all products are produced in a single tubular reactor. Moreover, all the products will be manufactured using optimal sequences (i.e. production wheels) to be determined as part of the solution of the SSC problem, where each sequence is repeated cyclically over an infinite time horizon (see Pinto and Grossmann [11] for the scheduling formulation). For modeling purposes the time is divided into a set of slots as depicted in Figure 1(a). Each slot is divided in a transition period and in a production period. As part of the solution of the SSC problem each slot features the production of a single product. Therefore, when a transition operation is enforced the operating conditions are changed from the old to the new product. Normally, for profit maximization product transitions should feature minimum transition time. During product transition the dynamic behavior of the system states and manipulated variables changes as a function of time. This behavior is conceptually represented in Figure 1(b). Once the system reaches the desired product, the production period starts and last until the production demands are met. It is worth to mention that when a production wheel is over then new identical production wheels start again.

Because the SSC optimization formulation used in this work is similar to a previously SSC formulation aimed to address continuous tank reactors [6], we have summarized the optimization formulation in the 


\section{Transition period}

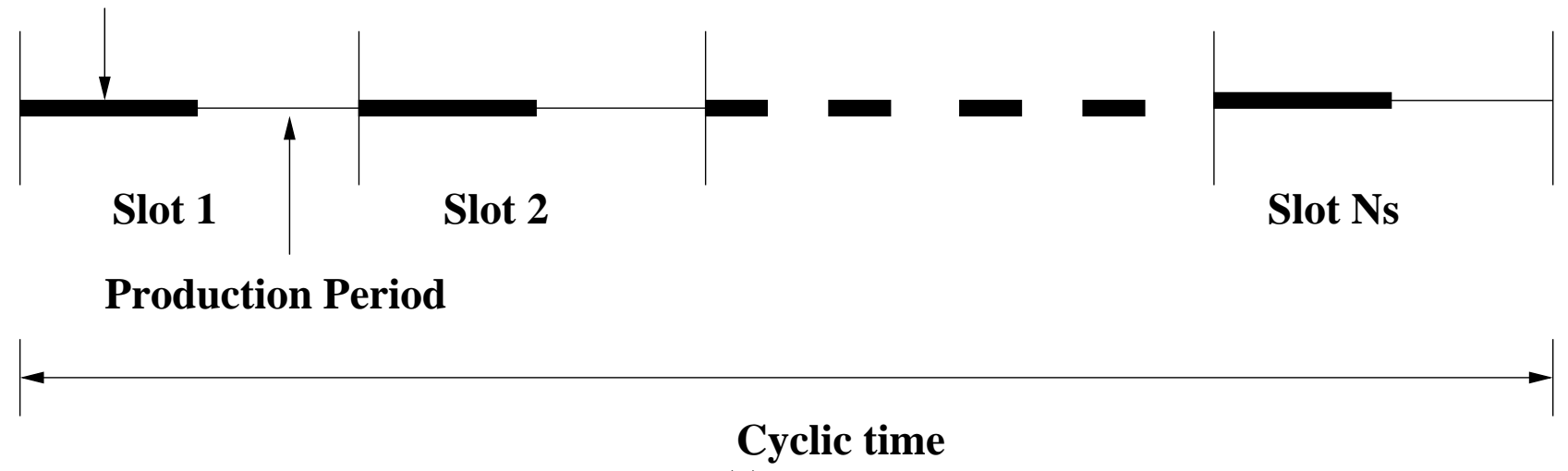

(a)

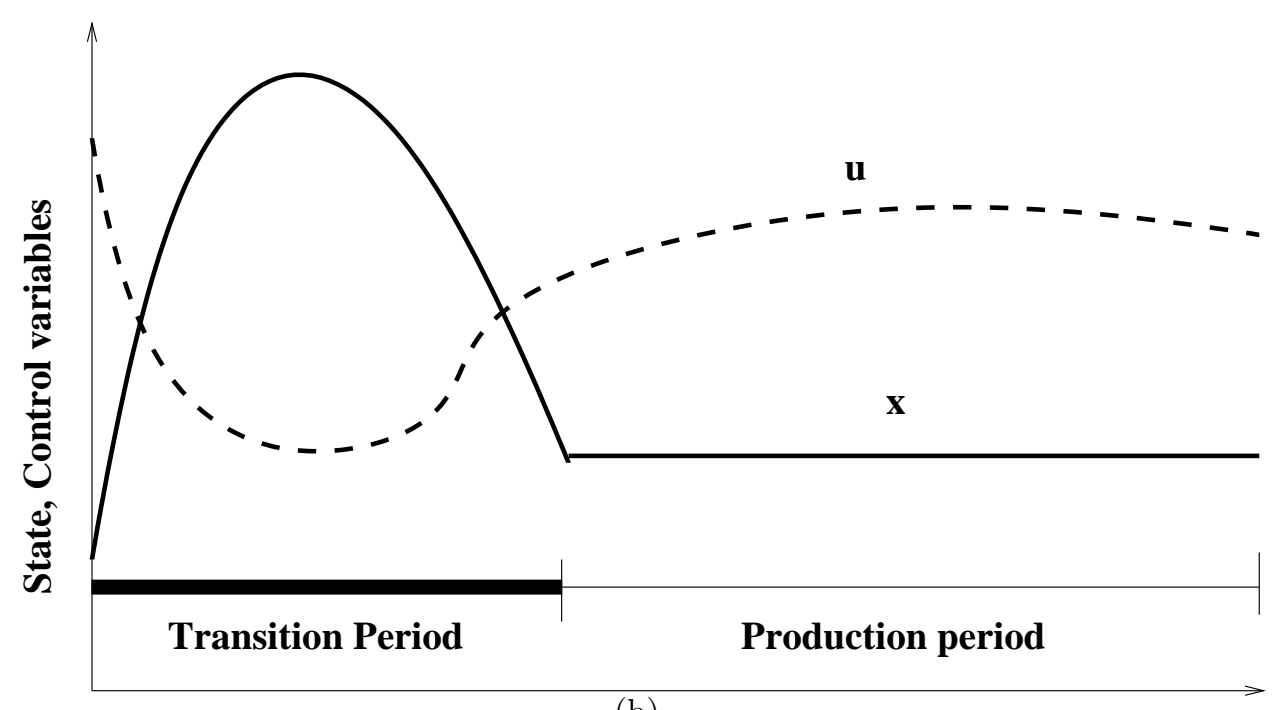

(b)

Figure 1: (a) At each production period the cyclic time is divided into $N_{s}$ slots. (b) When a change in the set-point of the system occurs there is a transition period followed by a continuous production period.

appendix section. This material has been included only for completeness. It is worth to mention that the only difference between the optimization formulation used in [6] and in the present work lies in the specific type of dynamic system. In [6] the dynamic system is composed by a system of ordinary differential equations, whereas in the present work the dynamic mathematical models are composed of a set of partial differential equations. Once the partial differential system is spatially discretized both optimization formulations become identical. 


\section{Model Spatial Discretization}

In this part, the method of lines was used for the discretization of the spatial partial derivatives [8]. The aim of such a transformation is to replace the spatial partial derivatives by a set of finite difference equations reducing the original PDE model to a set of ODEs. Hence, the resulting set of ODEs can be numerically integrated by standard ODEs numerical procedures. In this way both the steady-state and dynamic behaviour of the original PDE model can be obtained. Although there are some other well established numerical discretization techniques, such as the finite element [12] and spectral methods [13], the method of lines is easier to apply to the solution of complex PDE models.

The discretization procedures used in the method of lines are finite difference equations of different degrees and precision whose aim is to approximate the spatial behavior. The discretization can be done by using either a fixed or variable domain partition. Because discretization formulas based on fixed domain partitions are easier to use, in this part we will only use this type of discretization formulas. Mathematical

models of tubular reactors are characterized by featuring diffusive $\left(\frac{\partial^{2} C}{\partial x^{2}}\right)$, convective $\left(\frac{\partial C}{\partial x}\right)$ and reaction terms. Hence, PDE models containing diffusive, convective and reaction terms are termed either diffusionconvection-reaction or advection-convection-reaction models models.

Taking into account only the diffusive term, the model is classified as a PDE equation of parabolic type, whereas if only the convective term is considered, then the PDE equation is classified as of a hyperbolic type [8]. Therefore, the model of the tubular reactor featuring diffusion and convection terms is named a PDE model of parabolic-hyperbolic type. Moreover, this PDE model classification highlights the fact that different type of finite elements approximations are required for either parabolic or hyperbolic terms. Next, the discretization formulas for each type of terms are discussed. For this purpose, the spatial domain has been divided as depicted in Figure 2, In this figure $n$ stands for the total number of equally spaced discrete points, $x_{l}$ and $x_{u}$ are the minimum and maximum values of the spatial coordinate $x$, whereas $\Delta x$ is the constant distance between any two adjacent discrete points.

In order to compute a numerical solution to a second degree PDE model, two independent boundary conditions at certain locations of the reactor must be specified. In this work, Dirichlet $($ at $x=0)$ and 
Neumann (at $x=L$, where $L$ is the reactor length) boundary conditions are enforced at the inlet and outlet parts of the reactor, respectively. As shown below, the discretization formulas are different depending upon the type of boundary conditions, the spatial location of the discretization points, and whether the spatial derivatives define a parabolic or hyperbolic PDE term.

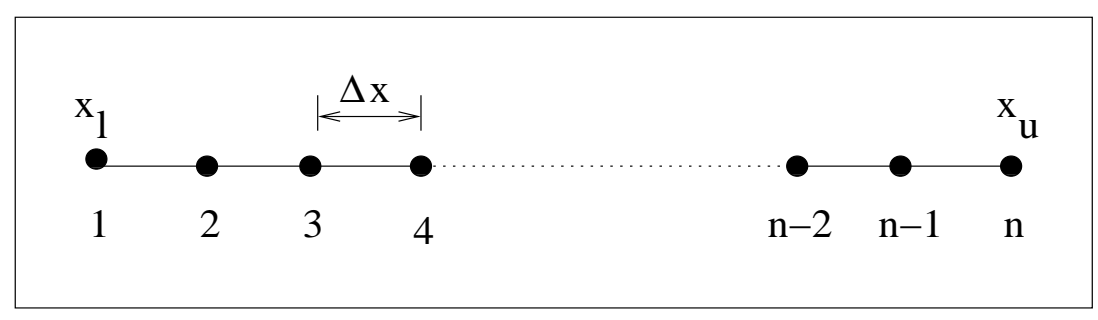

Figure 2: The spatial domain is divided into $n$ equally sized discretization intervals denoted by $\Delta x, x_{l}$ and $x_{u}$ are the lower and upper values of the spatial coordinate.

\section{- Diffusive term}

1. First point (Dirichlet boundary conditions)

$$
\left.\frac{\partial^{2} u}{\partial x^{2}}\right|_{1}=\beta\left(45 u_{1}-154 u_{2}+214 u_{3}-156 u_{4}+61 u_{5}-10 u_{6}\right)
$$

2. Interior grid points

$$
\begin{aligned}
\left.\frac{\partial^{2} u}{\partial x^{2}}\right|_{2} & =\beta\left(10 u_{1}-15 u_{2}-4 u_{3}+14 u_{4}-6 u_{5}+u_{6}\right) \\
\left.\frac{\partial^{2} u}{\partial x^{2}}\right|_{i} & =\beta\left(-u_{i-2}+16 u_{i-1}-30 u_{i}+16 u_{i+1}-u_{i+2}\right), i=3, \ldots, n-2 \\
\left.\frac{\partial^{2} u}{\partial x^{2}}\right|_{n-1} & =\beta\left(10 u_{n}-15 u_{n-1}-4 u_{n-2}+14 u_{n-3}-6 u_{n-4}+u_{n-5}\right)
\end{aligned}
$$

3. Last point (Neumann boundary conditions)

$$
\left.\frac{\partial^{2} u}{\partial x^{2}}\right|_{n}=\beta\left(-\frac{415}{6} u_{n}+96 u_{n-1}-36 u_{n-2}+\frac{32}{3} u_{n-3}-\frac{3}{2} u_{n-4}+\left.50 \frac{\partial u}{\partial x}\right|_{n} \Delta x\right)
$$


where,

$$
\begin{aligned}
\Delta x & =\frac{x_{u}-x_{l}}{n-1} \\
\beta & =\frac{1}{12 \Delta x^{2}}
\end{aligned}
$$

\section{- Convective term}

1. First point

$$
\left.\frac{\partial u}{\partial x}\right|_{1}=\gamma\left(-25 u_{1}+48 u_{2}-36 u_{3}+16 u_{4}-3 u_{5}\right)
$$

2. Interior grid points

$$
\begin{aligned}
& \left.\frac{\partial u}{\partial x}\right|_{2}=\gamma\left(-3 u_{1}-10 u_{2}+18 u_{3}-6 u_{4}+u_{5}\right) \\
& \left.\frac{\partial u}{\partial x}\right|_{3}=\gamma\left(u_{1}-8 u_{2}+8 u_{4}-u_{5}\right) \\
& \left.\frac{\partial u}{\partial x}\right|_{i}=\gamma\left(-u_{i-3}+6 u_{i-2}-18 u_{i-1}+10 u_{i}+3 u_{i+1}\right), i=4, \ldots, n-1
\end{aligned}
$$

3. Last point

$$
\left.\frac{\partial u}{\partial x}\right|_{n}=\gamma\left(3 u_{n-4}-16 u_{n-3}+36 u_{n-2}-48 u_{n-1}+25 u_{n}\right)
$$

where,

$$
\gamma=\frac{1}{12 \Delta x}
$$

\section{Case studies}

In this section several simultaneous scheduling and control problems taking place in tubular reactors are addressed. The examples were selected to feature different degrees of steady-state and dynamic nonlinear behavior, such that the complexity of solving scheduling and control problems is highlighted. We also did so hoping to justify the use of advanced decomposition optimization techniques such as Lagrangian decomposition [14] to address the scheduling and control of more complex reaction system such as polymerization 
reaction systems.

It is a well known fact that most NLP solvers require good initialization strategies to succeed in finding an optimal solution. This is especially true when the size and model complexity are factors that deserve careful consideration. In fact, if good initial guesses are not provided, finding even a feasible solution can be hard to achieve. In our experience, the scheduling and control optimization of distributed parameter systems can be efficiently tackled by decomposing the original complex problem into a series of simpler subproblems. The idea is to use the simpler subproblems to initialize more complex versions of the problem to be solved. In this work, we use the natural decomposition present in scheduling and control problems to solve simultaneous scheduling and control problems in distributed parameter systems. The approach is as follows:

1. Solve the scheduling problem by fixing transition times.

2. Compute steady-state processing conditions for the set of products to be manufactured.

3. Solve the dynamic optimization problem by fixing the best production sequence found in step (1) above.

4. Solve the simultaneous scheduling and control problem by relaxing the computation of the best production sequence and optimal product transitions.

As indicated, the idea behind the initialization procedure is to use results from a previous step to initialize the next one. All the simultaneous scheduling and control problems were solved using the above solution strategy. For the numerical solution of the MINLP problem arising from the discretization of the original MIDO problem the sbb/CONOPT MINLP solver in gams [15] was used. After some trials, we came to the conclusion that 11 equal sized points were enough for spatial discretization, whereas 20 finite elements and 3 internal collocation points sufficed for time discretization. Moreover, all the problems were solved using 2 Ghz, 4 Gb computer. 


\section{Isothermal tubular reactor}

The reaction $2 A \rightarrow B$ takes place in a continuous tubular reactor. For modeling this reaction system we assume isothermal conditions, plug flow conditions and mass transfer along the longitudinal direction. Both diffusive and convective mass transfer contributions are also modeled. Under these operating conditions the one-dimensional dynamic mathematical model of the tubular reactor reads as follows:

$$
\frac{\partial C}{\partial t}=D \frac{\partial^{2} C}{\partial x^{2}}-v \frac{\partial C}{\partial x}-K C^{2}
$$

subject to the following initial,

$$
C(x, 0)=C_{f}
$$

and boundary conditions,

$$
\begin{array}{ll}
\text { @ } x=0 & C=C_{f}+\frac{D}{v} \frac{\partial C}{\partial x} \\
@ x=L & \frac{\partial C}{\partial x}=0
\end{array}
$$

where $C$ stands for the concentration of the $A$ component, $x$ is the longitudinal coordinate, $t$ is the time, $D$ is the mass diffusivity and $v$ is the linear velocity, $K$ is the reaction constant, $C_{f}$ is the feed stream composition of reactant $A$ and $L$ is the reactor length. The above model can be cast in dimensionless form by using the following dimensionless variables:

$$
C^{\prime}=\frac{C}{C_{f}}, \quad x^{\prime}=\frac{x}{L}, \quad \theta=\frac{t v}{L}
$$

Hence, in terms of the dimensionless variables, the scaled model reads as follows,

$$
\frac{\partial C^{\prime}}{\partial \theta}=\frac{1}{P e_{M}} \frac{\partial^{2} C^{\prime}}{\partial x^{\prime 2}}-\frac{\partial C^{\prime}}{\partial x^{\prime}}-\frac{\alpha}{P e_{M}} C^{\prime 2}
$$




\begin{tabular}{|cccccccccc|}
\hline Product & $\begin{array}{c}\text { Conversion } \\
\text { fraction }\end{array}$ & $\begin{array}{c}\text { Demand } \\
\text { rate }[\mathrm{Kg} / \mathrm{h}]\end{array}$ & $\begin{array}{c}\text { Process } \\
\text { cost }[\$ / \mathrm{kg}]\end{array}$ & $\begin{array}{c}\text { Inventory } \\
\text { cost }[\$ / \mathrm{kg}]\end{array}$ & \multicolumn{4}{c}{ Transition Cost $[\$]$} \\
\hline$A$ & 0.5024 & 100 & 620 & 1.5 & 0 & 350 & 700 & 600 & 400 \\
$B$ & 0.6036 & 90 & 730 & 1 & 400 & 0 & 550 & 450 & 500 \\
$C$ & 0.6959 & 120 & 750 & 2 & 800 & 600 & 0 & 550 & 800 \\
$D$ & 0.8019 & 80 & 770 & 3 & 500 & 400 & 600 & 0 & 700 \\
$E$ & 0.9 & 70 & 790 & 2.5 & 600 & 370 & 550 & 700 & 0 \\
\hline
\end{tabular}

Table 1: Process data for the isothermal tubular reactor case study. $A, B, C, D$ and $E$ stand for the five products to be manufactured.

subject to the following initial,

$$
C^{\prime}\left(x^{\prime}, 0\right)=1
$$

and dimensionless boundary conditions,

$$
\begin{array}{ll}
@ x=0 & \frac{\partial C^{\prime}}{\partial x^{\prime}}=P e_{M}\left(C^{\prime}-1\right) \\
@ x=1 & \frac{\partial C^{\prime}}{\partial x^{\prime}}=0
\end{array}
$$

where $P e_{M}=\frac{L v}{D}$ is the mass transfer Peclet number and $\alpha=\frac{L^{2} K C_{f}}{D}$. Using $L=20 \mathrm{~m}, D=10 \mathrm{~m}^{2} / \mathrm{s}$, $C_{f}=100 \mathrm{kmol} / \mathrm{m}^{3}, r=0.5 \mathrm{~m}$ and $K=1 \times 10^{-3} \mathrm{~m}^{3} / \mathrm{kmol}-\mathrm{h}$, lead to the production of five products $A, B, C, D$ and $E$ which are defined in Table 1. In addition, Table 1 contains information regarding the cost of the products, demand rate, inventory cost, and production rate of each one of the hypothetical products. The manipulated variable for optimal product transitions is the volumetric flow rate.

The optimal production sequence resulting from the simultaneous scheduling and control problem turns out to be: $D \rightarrow B \rightarrow A \rightarrow E \rightarrow C$, the duration time of the cyclic production sequence is $252.031 \mathrm{~h}$, featuring a profit of $\$ 1.013 \times 10^{6}$. The optimal solution was obtained in 55 min CPU time. The number of equations, continuous and discrete variables is 14846, 15217 and 50, respectively. In Table 2, a summary of the results of the optimal solution is shown, whereas the optimal dynamic transition profiles among the manufactured products are depicted in Figure 3, We should note that this solution is not guaranteed to be globally optimal due to the nonconvexities in the MINLP model. 


\begin{tabular}{|cccccccccc|}
\hline Slot & Prod. & $\begin{array}{c}\text { Volumetric } \\
\text { Flow }\left[\mathrm{m}^{3} / \mathrm{s}\right]\end{array}$ & $\begin{array}{c}\text { Conc. } \\
{\left[\mathrm{kmol} / \mathrm{m}^{3}\right]}\end{array}$ & $\begin{array}{c}\theta \\
{[\mathrm{h}]}\end{array}$ & $\begin{array}{c}\text { Amount } \\
\text { Produced }[\mathrm{Kg}]\end{array}$ & $\begin{array}{c}\text { Production } \\
\text { rate }[\mathrm{kg} / \mathrm{h}]\end{array}$ & $\begin{array}{c}\text { Process } \\
\text { time }[\mathrm{h}]\end{array}$ & $\begin{array}{c}\text { T start } \\
{[\mathrm{h}]}\end{array}$ & $\begin{array}{c}\text { T end } \\
{[\mathrm{h}]}\end{array}$ \\
\hline 1 & $D$ & 0.1 & 19.8 & 2 & 20162.489 & 762.872 & 26.43 & 0 & 31.43 \\
2 & $B$ & 0.62 & 39.6 & 2.5 & 22682.801 & 3555.098 & 6.38 & 31.43 & 42.81 \\
3 & $A$ & 1.169 & 49.8 & 0.5 & $4.2360 \times 10^{5}$ & 5580.029 & 75.91 & 42.81 & 123.72 \\
4 & $E$ & 0.02 & 10 & 1.5 & 17642.178 & 170.978 & 103.18 & 123.72 & 231.9 \\
5 & $C$ & 0.302 & 30.4 & 0.5 & 30243.734 & 1999.680 & 15.12 & 231.9 & 252.03 \\
\hline
\end{tabular}

Table 2: Simultaneous Scheduling and Control results for the isothermal tubular reactor case study. The best production sequence is: $D \rightarrow B \rightarrow A \rightarrow E \rightarrow C$ featuring $252.031 \mathrm{~h}$ as total cyclic time and objective function value of $\$ 1.013 \times 10^{6}$. The optimal solution was computed in 55 min CPU time. $\theta$ stands for discretized transition times.

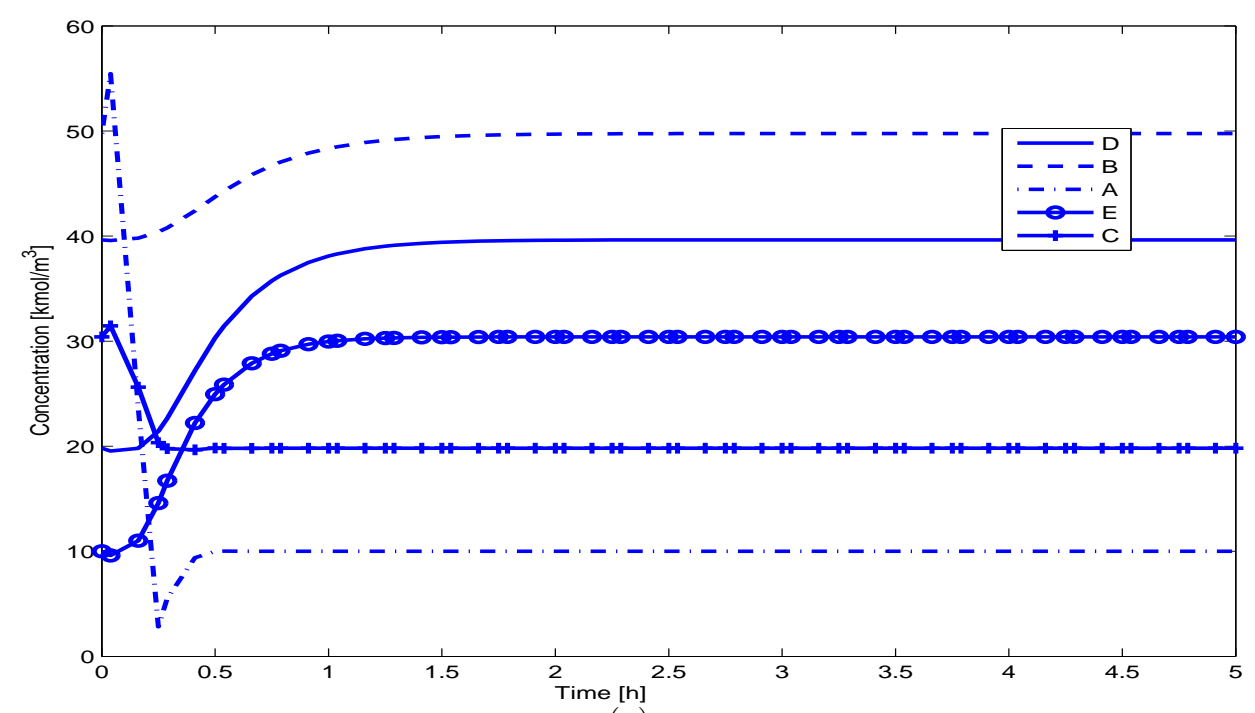

(a)

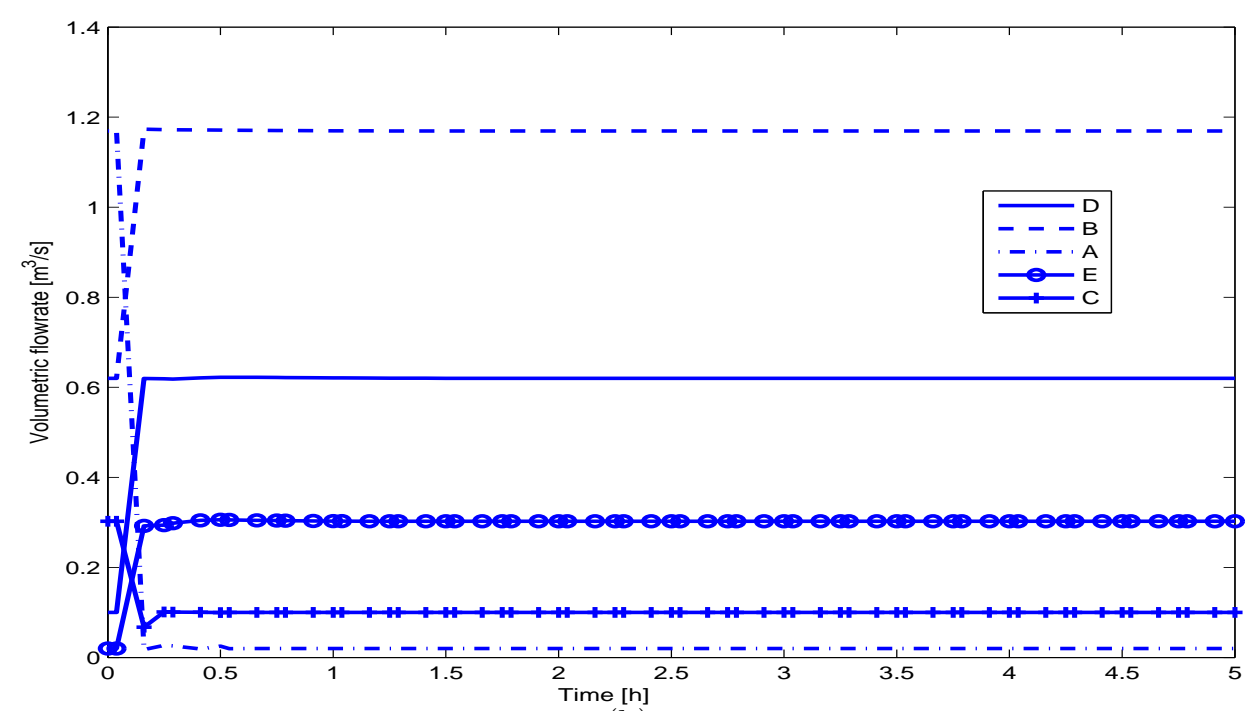

(b)

Figure 3: Optimal dynamic transition profiles for the isothermal tubular reactor. The best production sequence is: $D \rightarrow B \rightarrow A \rightarrow E \rightarrow C$. 
It should be pointed out that there are other optimal production sequences that are equivalent to the one found previously. This means that these optimal sequences feature the same objective function value, cyclic time, amount produced, production rate, processing time, etc. For instance, the sequences $B \rightarrow$ $A \rightarrow E \rightarrow C \rightarrow D$ and $A \rightarrow E \rightarrow C \rightarrow D \rightarrow B$ are equivalent to the aforementioned optimal production sequence. Due to the cyclic production wheel assumption, it is completely irrelevant whether product $D$ is actually manufactured at the first, second, third, fourth or fifth slot. All the equivalent optimal production sequences feature the same production order. However, we have found that equivalent optimal sequences tend to demand different CPU times for finding an optimal solution.

Regarding the optimal dynamic transition profiles, as depicted in Figure 3, the system response is, in general, smooth. This is especially true when the product transition involves reducing product conversion as done in slots where products $D, B$ and $E$ are manufactured. When the product transition involves increasing product conversion, as in the slots where products $C$ and $A$ are manufactured, an inverse response is observed. In fact, this effect is more notorious for product $A$. However, at the end of the transition period, all the products attain the final steady-state operating conditions. On the other hand, the manipulated variable assumes ramp-like changes with large slopes. Looking at Figure 3 , the reasons of the aforementioned apparent system inverse response behavior become clear. In the case of products $D$, $B$ and $E$, the manipulated variable response takes a ramp-like shape without changes in the slope sign. However, in those cases where an inverse response is observed, products $C$ and $A$, there are some changes in the sign of the slope during the product transition. It may be argued whether by using simple step changes in the manipulated variable, one could get similar results to the ones shown in Figure 3. This may be apparent after the fact, but it is hard to guess before carrying out all the optimal dynamic transition calculations. Anyway, our results feature optimality characteristics, a point that cannot be raised in favor of simple step changes. 


\section{Nonisothermal plug-flow tubular reactor}

Let us consider the nonisothermal operation of a one-dimensional tubular reactor where the set of reactions is given by:

$$
A \stackrel{k_{10}}{\rightarrow} B \stackrel{k_{20}}{\rightarrow} C
$$

By assuming plug-flow, negligible temperature and concentration radial variations and no diffusion along the axial spatial coordinate, the distributed mathematical model of the systems reads as follows [16]:

$$
\begin{aligned}
\frac{\partial C_{A}}{\partial t}= & -v \frac{\partial C_{A}}{\partial x}-k_{10} e^{-E_{1} / R T_{r}} C_{A} \\
\frac{\partial C_{B}}{\partial t}= & -v \frac{\partial C_{B}}{\partial x}+k_{10} e^{-E_{1} / R T_{r}} C_{A}-k_{20} e^{-E_{2} / R T_{r}} C_{B} \\
\frac{\partial T_{r}}{\partial t}= & -v \frac{\partial T_{r}}{\partial x}+\frac{\left(-\Delta H_{R_{A}}\right)}{\rho_{m} C_{p m}} k_{10} e^{-E_{1} / R T_{r}} C_{A} \\
& -\frac{\left(-\Delta H_{R_{B}}\right)}{\rho_{m} C_{p m}} k_{20} e^{-E_{2} / R T_{r}} C_{B}+\frac{U_{w} A}{\rho_{m} C_{p m} V_{r}}\left(T_{j}-T_{r}\right)
\end{aligned}
$$

where $C_{A}$ is the concentration of component $A, C_{B}$ is the concentration of component $B, T_{r}$ is the reactor temperature, $t$ is stands for time, $x$ denotes the length of the reactor and $v$ is the linear velocity. The meaning of the rest of the variables and their corresponding numerical values are shown in Table 3 . The aforementioned model is subject to the following initial

$$
\begin{aligned}
\left.C_{A}\right|_{x, t=0} & =C_{A}^{s} \\
\left.C_{B}\right|_{x, t=0} & =C_{B}^{s} \\
\left.T_{r}\right|_{x, t=0} & =T_{r}^{s}
\end{aligned}
$$

and boundary conditions:

$$
\begin{gathered}
\left.C_{A}\right|_{x=0, t}=C_{A}^{f} \\
\left.C_{B}\right|_{x=0, t}=C_{B}^{f} \\
\left.T_{r}\right|_{x=0, t}=T_{r}^{f}
\end{gathered}
$$


where the superscripts $s, f$ stand for steady-state processing conditions and feed stream conditions, respectively. By defining the following dimensionless variables:

$$
\begin{aligned}
\hat{C}_{A} & =\frac{C_{A}}{C_{A}^{f}} \\
\hat{C}_{B} & =\frac{C_{B}}{C_{A}^{f}} \\
\hat{T}_{r} & =\frac{T_{r}}{T_{r}^{f}} \\
\hat{T}_{j} & =\frac{T_{j}}{T_{r}^{f}} \\
\hat{x} & =\frac{x}{L} \\
\theta & =\frac{t v}{L}
\end{aligned}
$$

the dimensionless PDE model reads as follows:

$$
\begin{aligned}
\frac{\partial \hat{C}_{A}}{\partial \hat{\theta}}= & -\frac{\partial \hat{C}_{A}}{\partial \hat{x}}-\frac{k_{10} L}{v} e^{-E_{1} / R T_{r}^{f} \hat{T}_{r}} \hat{C}_{A} \\
\frac{\partial \hat{C}_{B}}{\partial \hat{\theta}}= & -\frac{\partial \hat{C}_{B}}{\partial \hat{x}}+\frac{k_{10} L}{v} e^{-E_{1} / R T_{r}^{f} \hat{T}_{r}} \hat{C}_{A}-\frac{k_{20} L}{v} e^{-E_{2} / R T_{r}^{f} \hat{T}_{r}} \hat{C}_{B} \\
\frac{\partial \hat{T}_{r}}{\partial \hat{\theta}}= & -\frac{\partial \hat{T}_{r}}{\partial \hat{x}}+\frac{\left(-\Delta H_{R_{A}} k_{10} C_{A}^{f}\right)}{\rho_{m} C_{p m} T_{r}^{f} v} e^{-E_{1} / R T_{r}^{f} \hat{T}_{r}} \hat{C}_{A} \\
& -\frac{\left(-\Delta H_{R_{B}} k_{20} C_{A}^{f} L\right)}{\rho_{m} C_{p m} T_{r}^{f} v} e^{-E_{2} / R T_{r}^{f} \hat{T}_{r}} \hat{C}_{B}+\frac{U_{w} A L}{\rho_{m} C_{p m} V_{r} v}\left(\hat{T}_{j}-\hat{T}_{r}\right)
\end{aligned}
$$

and the corresponding initial

$$
\begin{aligned}
\left.\hat{C}_{A}\right|_{\hat{x}, \theta=0} & =\frac{C_{A}^{s}}{C_{A}^{f}} \\
\left.\hat{C}_{B}\right|_{\hat{x}, \theta=0} & =\frac{C_{B}^{s}}{C_{A}^{f}} \\
\left.\hat{T}_{r}\right|_{\hat{x}, \theta=0} & =\frac{T_{r}^{s}}{T_{r}^{f}}
\end{aligned}
$$




\begin{tabular}{|llll|}
\hline Variable & Description & Value & Units \\
\hline$L$ & reactor length & 1 & $\mathrm{~m}$ \\
$E_{1}$ & activation energy first reaction & 20000 & $\mathrm{kcal} / \mathrm{gmol}$ \\
$E_{2}$ & activation energy second reaction & 50000 & $\mathrm{kcal} / \mathrm{kgmol}$ \\
$k_{10}$ & pre-exponential factor first reaction & $5 \times 10^{12}$ & $1 / \mathrm{min}$ \\
$k_{20}$ & pre-exponential factor second reaction & $5 \times 10^{12}$ & $1 / \mathrm{min}$ \\
$\Delta H_{R_{A}}$ & heat of reaction of first reaction & -0.548 & $\mathrm{kcal} / \mathrm{kgmol}$ \\
$\Delta H_{R_{B}}$ & heat of reaction of second reaction & -0.986 & $\mathrm{kcal} / \mathrm{kgmol}$ \\
$\rho_{m}$ & density of reacting mixture & 0.09 & $\mathrm{~kg} / \mathrm{L}$ \\
$A$ & heat transfer area & 1 & $\mathrm{~m} 2$ \\
$R$ & ideal gas constant & 1.987 & $\mathrm{kcal} / \mathrm{kgmol}-\mathrm{K}$ \\
$U_{w}$ & heat transfer coefficient & 2 & $\mathrm{kcal} / \mathrm{m}^{2}-\mathrm{K}-\mathrm{min}$ \\
$C_{p m}$ & reacting mixture heat capacity & 0.231 & $\mathrm{kcal} / \mathrm{kg}-\mathrm{K}$ \\
$M_{w_{A}}$ & molecular weight compound A & 200 & $\mathrm{~kg} / \mathrm{kmol}$ \\
$M_{w_{B}}$ & molecular weight compound B & 190 & $\mathrm{~kg} / \mathrm{kmol}$ \\
$C_{A}^{f}$ & reactant A feed stream composition & $4 \times 10^{-3}$ & $\mathrm{Kmol} / \mathrm{L}$ \\
$C_{B}^{f}$ & product B feed steam composition & 0 & $\mathrm{Kmol} / \mathrm{L}$ \\
$T_{r}^{f}$ & reactor feed stream temperature & 320 & $\mathrm{~K}$ \\
$T_{j}$ & cooling fluid stream temperature & 352 & $\mathrm{~K}$ \\
$V_{r}$ & reactor volume & 10 & $\mathrm{~L}$ \\
\hline
\end{tabular}

Table 3: Design data for the nonisothermal plug-flow tubular reactor case study.

and boundary dimensionless conditions:

$$
\begin{aligned}
\left.\hat{C}_{A}\right|_{\hat{x}=0, \theta} & =1 \\
\left.\hat{C}_{B}\right|_{\hat{x}=0, \theta} & =1 \\
\left.\hat{T}_{r}\right|_{\hat{x}=0, \theta} & =1
\end{aligned}
$$

Information regarding the desired products, conversion fraction, demand, cost of the products and inventory cost are shown in Table 4. The variable used as manipulated variable for optimal product transitions is the linear velocity $v$.

The optimal production sequence resulting from the simultaneous scheduling and control problem turns out to be: $A \rightarrow B \rightarrow C \rightarrow E \rightarrow D$, the duration time of the cyclic production sequence is $137.598 \mathrm{~h}$, featuring a profit of $\$ 22816.78$. The optimal solution was obtained in $1.15 \mathrm{~h}$ CPU time. The number of equations, continuous and discrete variables is 33566, 37357 and 50, respectively. In Table 5, a summary 


\begin{tabular}{|c|c|c|c|c|c|c|c|c|c|}
\hline Product & Conversion & Demand & Process & Inventory & & ansit & on ( & ost & $\$]$ \\
\hline & Fraction & rate $[\mathrm{Kg} / \mathrm{m}]$ & cost $[\$ / \mathrm{kg}]$ & cost $[\$ / \mathrm{kg}]$ & $A$ & $B$ & $C$ & $D$ & $E$ \\
\hline $\bar{A}$ & 0.5 & 6 & 150 & 1.5 & 0 & 5 & 6 & 7 & 8 \\
\hline$B$ & 0.6 & 8 & 200 & 1 & 10 & 0 & 5 & 8 & 6 \\
\hline C & 0.7 & 7 & 240 & 2 & 7 & 10 & 0 & 8 & 7 \\
\hline$D$ & 0.8 & 6 & 280 & 3 & 6 & 10 & 10 & 0 & 12 \\
\hline$E$ & 0.9 & 5 & 320 & 2.5 & 8 & 9 & 10 & 10 & 0 \\
\hline
\end{tabular}

Table 4: Process data for the non-isothermal plug-flow tubular reactor case study. $A, B, C, D$ and $E$ stand for the five products to be manufactured.

of the results of the optimal solution are shown, whereas the optimal dynamic transition profiles among the manufactured products are depicted in Figure 4 .

\begin{tabular}{|c|c|c|c|c|c|c|c|c|c|c|c|}
\hline Slot & Prod. & $\hat{C}_{A}$ & $\hat{C}_{B}$ & $\hat{T}_{r}$ & $\begin{array}{c}v \\
{[\mathrm{~m} / \mathrm{min}]}\end{array}$ & $\begin{array}{c}\theta \\
{[\mathrm{h}]}\end{array}$ & $\begin{array}{c}\text { Amount } \\
\text { Produced }[\mathrm{Kg}]\end{array}$ & $\begin{array}{l}\text { Production } \\
\text { rate }[\mathrm{kg} / \mathrm{m}]\end{array}$ & $\begin{array}{l}\text { Process } \\
\text { time }[\mathrm{h}]\end{array}$ & $\begin{array}{c}\text { T start } \\
{[\mathrm{h}]}\end{array}$ & $\begin{array}{c}\mathrm{T} \text { end } \\
{[\mathrm{h}]}\end{array}$ \\
\hline 1 & $\bar{A}$ & 0.5 & 0.5 & 1.151 & 4.269 & 2 & 825.59 & 129.115 & 6.4 & 0 & 11.39 \\
\hline 2 & $B$ & 0.4 & 0.6 & 1.155 & 3.765 & 1.8 & 1100 & 136.674 & 8.1 & 11.39 & 24.44 \\
\hline 3 & C & 0.3 & 0.7 & 1.159 & 3.309 & 1.6 & 963.188 & 140.129 & 6.8 & 24.44 & 36.32 \\
\hline 4 & $E$ & 0.1 & 0.9 & 1.166 & 2.858 & 2.1 & 10896.744 & 127.734 & 85.3 & 36.32 & 126.63 \\
\hline 5 & $D$ & 0.2 & 0.8 & 1.163 & 2.346 & 2.2 & 825.59 & 138.335 & 5.97 & 126.63 & 137.6 \\
\hline
\end{tabular}

Table 5: Simultaneous Scheduling and Control results for the non-isothermal plug flow tubular reactor case study. The best production sequence is: $A \rightarrow B \rightarrow C \rightarrow E \rightarrow D$ featuring $137.598 \mathrm{~h}$ as total cyclic time and objective function value of $\$ 22816.78$. The optimal solution was computed in $1.55 \mathrm{~h}$ CPU time. $\theta$ stands for transition times.

Regarding the optimal dynamic transition profiles shown in Figure 4, we note that all the system responses feature smooth behavior. In this case no system inverse response was observed. This is so because the manipulated variable takes a ramp-like shape without changing the sign of their slopes. Once again, one could be tempted to argue that no dynamic optimization calculations would be needed, if one were to just use simple step changes in the manipulated variables values. This, however, overlooks several important points. First, it is hard to guess the optimal shape of the manipulated variables response without actually calculating the optimal solution. Second, our calculations ensure (local) optimality conditions, whereas system response obtained by applying simple step-changes does not. Moreover, even when one could guess the shape of the manipulated variables behavior, it is hard to have good guesses of the optimal production sequence. Because of these reasons, instead of using a trial and error procedure, we claim that much better 
results are obtained by solving the scheduling and control problem by a simultaneous approach and within a formal optimization framework. Finally, it is worth to mention that the present case study demanded almost twice the CPU time of the first case study. The CPU time increased because of the larger number of continuous variables, since the present distributed model feature more system states. To keep the CPU time relatively low, we decided not to increase the number of integers variables. In the next case study, we will approach a similar distributed parameters system example, but featuring a larger number of integer variables, just to analyze the effect that increasing the number of binary decision variables has on the CPU time.

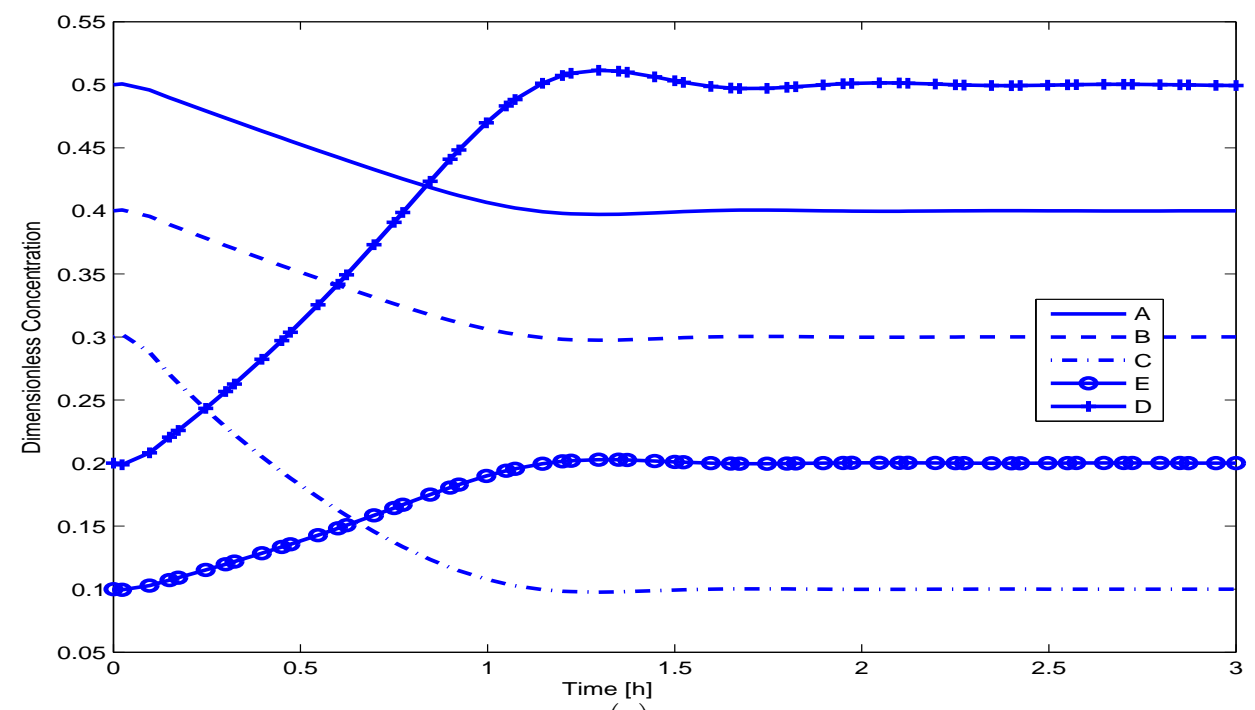

(a)

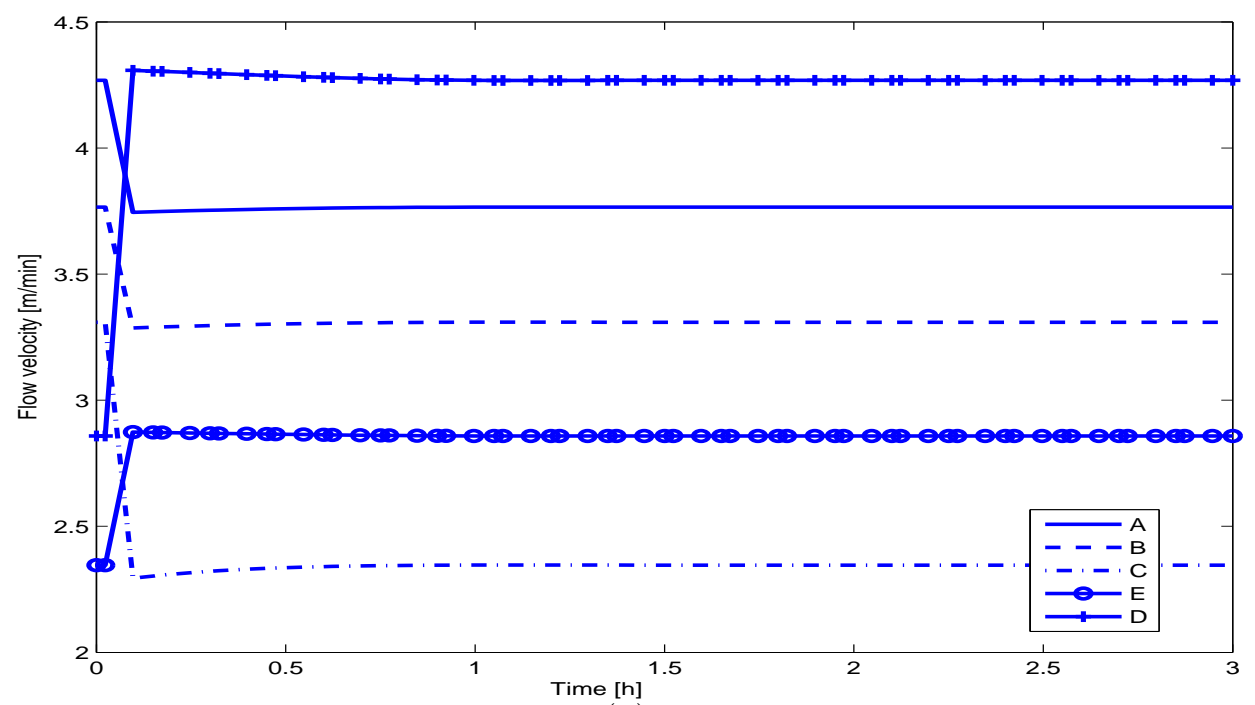

(b)

Figure 4: Optimal dynamic transition profiles for the non-isothermal plug-flow tubular reactor. The optimal production sequence is: $A \rightarrow B \rightarrow C \rightarrow E \rightarrow D$. 


\section{Nonisothermal catalytic fixed-bed tubular reactor}

In this example, we use the model of a nonisothermal catalytic fixed-bed tubular reactor. This model happens to display strong nonlinear behavior, and demands large CPU time for the computation of the simultaneous scheduling and control optimal solution. The distributed parameter model reads as follows:

$$
\begin{aligned}
\frac{\partial C_{A}}{\partial t}= & -\frac{v}{\epsilon} \frac{\partial C_{A}}{\partial x}+\frac{\mu(1-\epsilon)}{\epsilon}\left[-k_{1} e^{-E_{1} / R T} C_{A}-k_{3} e^{-E_{3} / R T} C_{A}\right] \\
\frac{\partial C_{B}}{\partial t}= & -\frac{v}{\epsilon} \frac{\partial C_{B}}{\partial x}+\frac{\mu(1-\epsilon)}{\epsilon}\left[k_{1} e^{-E_{1} / R T} C_{A}-k_{2} e^{-E_{2} / R T} C_{B}\right] \\
\frac{\partial T}{\partial t}= & -\frac{v}{L e} \frac{\partial T}{\partial x}+\frac{\mu(1-\epsilon)}{L e \rho_{f} C_{p f}}\left[-\Delta H_{r 1} k_{1} e^{-E_{1} / R T} C_{A}-\Delta H_{r 2} k_{2} e^{-E_{2} / R T} C_{B}\right. \\
& \left.-\Delta H_{r 3} k_{3} e^{-E_{3} / R T} C_{A}\right]+\frac{2 U L}{L e r^{\prime} \rho_{f} C_{p f}}\left(T_{w}-T\right)
\end{aligned}
$$

where $C_{A}$ is the concentration of component $A, C_{B}$ is the concentration of component $B, T$ is the reactor temperature, $t$ is stands for time, $x$ denotes the length of the reactor, $v$ is the linear velocity and $L e=$

$\frac{(1-\epsilon) \rho^{s} C_{p}^{s}+\epsilon \rho^{f} C_{p}^{f}}{\rho^{f} C_{p c}}$ is the Lewis number. The meaning of the rest of the variables, and their corresponding numerical values are shown in Table 6. The aforementioned model is subject to the following initial

$$
\begin{aligned}
\left.C_{A}\right|_{x, t=0} & =C_{A}^{s} \\
\left.C_{B}\right|_{x, t=0} & =C_{B}^{s} \\
\left.T\right|_{x, t=0} & =T^{s}
\end{aligned}
$$


and boundary conditions:

$$
\begin{aligned}
\left.C_{A}\right|_{x=0, t} & =C_{A}^{f}+\frac{D_{m}}{v} \frac{\partial C_{A}}{\partial x} \\
\left.C_{B}\right|_{x=0, t} & =C_{B}^{f}+\frac{D_{m}}{v} \frac{\partial C_{B}}{\partial x} \\
\left.T\right|_{x=0, t} & =T^{f}+\frac{D_{t}}{v} \frac{\partial T}{\partial x} \\
\left.\frac{\partial C_{A}}{\partial x}\right|_{x=L, t} & =0 \\
\left.\frac{\partial C_{B}}{\partial x}\right|_{x=L, t} & =0 \\
\left.\frac{\partial T}{\partial x}\right|_{x=L, t} & =0
\end{aligned}
$$

where the superscript $s$ stands for steady-state processing conditions. By defining the following dimensionless variables:

$$
\begin{aligned}
\hat{C}_{A} & =\frac{C_{A}}{C_{A}^{f}} \\
\hat{C}_{B} & =\frac{C_{B}}{C_{B}^{f}} \\
\hat{T} & =\frac{T}{T^{f}} \\
\hat{T}_{w} & =\frac{T_{w}}{T^{f}} \\
\hat{x} & =\frac{x}{L} \\
\theta & =\frac{t v}{L}
\end{aligned}
$$

the dimensionless PDE model reads as follows:

$$
\begin{aligned}
\frac{\partial \hat{C}_{A}}{\partial \theta}= & -\frac{1}{\epsilon} \frac{\partial \hat{C}_{A}}{\partial \hat{x}}+\frac{L \mu(1-\epsilon)}{v \epsilon}\left[-k_{1} e^{-E_{1} / R T^{f} \hat{T}} \hat{C}_{A}-k_{3} e^{-E_{3} / R T^{f} \hat{T}} \hat{C}_{A}\right] \\
\frac{\partial \hat{C}_{B}}{\partial \theta}= & -\frac{1}{\epsilon} \frac{\partial \hat{C}_{B}}{\partial \hat{x}}+\frac{L \mu(1-\epsilon)}{v \epsilon}\left[k_{1} e^{-E_{1} / R T^{f} \hat{T}} \hat{C}_{A}-k_{2} e^{-E_{2} / R T^{f} \hat{T}} \hat{C}_{B}\right] \\
\frac{\partial \hat{T}}{\partial \theta}= & -\frac{1}{L e} \frac{\partial \hat{T}}{\partial \hat{x}}+\frac{\mu(1-\epsilon) C_{A}^{f} L}{L e \rho_{f} C_{p f} v T^{f}}\left[-\Delta H_{r 1} k_{1} e^{-E_{1} / R T^{f} \hat{T}} \hat{C}_{A}-\Delta H_{r 2} k_{2} e^{-E_{2} / R T^{f} \hat{T}} \hat{C}_{B}\right. \\
& \left.-\Delta H_{r 3} k_{3} e^{-E_{3} / R T^{f} \hat{T}} \hat{C}_{A}\right]+\frac{2 U L^{2}}{L e r^{\prime} \rho_{f} C_{p f} v}\left(\hat{T}_{w}-\hat{T}\right)
\end{aligned}
$$


and the initial:

$$
\begin{aligned}
\left.\hat{C}_{A}\right|_{\hat{x}, \theta=0} & =\frac{C_{A}^{s}}{C_{A}^{f}} \\
\left.\hat{C}_{B}\right|_{\hat{x}, \theta=0} & =\frac{C_{B}^{s}}{C_{B}^{f}} \\
\left.\hat{T}\right|_{\hat{x}, \theta=0} & =\frac{T^{s}}{T^{f}}
\end{aligned}
$$

and boundary dimensionless conditions:

$$
\begin{aligned}
\left.\hat{C}_{A}\right|_{\hat{x}=0, \theta} & =1+\frac{D_{m}}{v L} \frac{\partial \hat{C}_{A}}{\partial \hat{x}} \\
\left.\hat{C}_{B}\right|_{\hat{x}=0, \theta} & =1+\frac{D_{m}}{v L} \frac{\partial \hat{C}_{B}}{\partial \hat{x}} \\
\left.\hat{T}\right|_{\hat{x}=0, \theta} & =1+\frac{D_{t}}{v L} \frac{\partial \hat{T}}{\partial \hat{x}} \\
\left.\frac{\partial \hat{C}_{A}}{\partial \hat{x}}\right|_{\hat{x}=1, \theta} & =0 \\
\left.\frac{\partial \hat{C}_{B}}{\partial \hat{x}}\right|_{\hat{x}=1, \theta} & =0 \\
\left.\frac{\partial \hat{T}}{\partial \hat{x}}\right|_{\hat{x}=1, \theta} & =0
\end{aligned}
$$

Information regarding the desired products, conversion fraction, demand, cost of the products and inventory cost is shown in Table 7. The variable used as manipulated variable for optimal product transitions is the volumetric flow rate.

The optimal production sequence resulting from the simultaneous scheduling and control problem turns out to be: $A \rightarrow B \rightarrow E \rightarrow F \rightarrow D \rightarrow C$, the duration time of the cyclic production sequence is 1479.119 h, featuring a profit of $\$ 787919.947$. The optimal solution was obtained in $12.08 \mathrm{~h}$ CPU. The number of equations, continuous and discrete decision variables is 40357, 40952 and 72, respectively. In Table 8, a summary of the results about the local optimal solution are shown, whereas the optimal dynamic transition profiles among the manufactured products are depicted in Figure 5. 


\begin{tabular}{|llll|}
\hline Variable & Description & Value & Units \\
\hline$L$ & reactor length & 4 & $\mathrm{~m}$ \\
$\epsilon$ & pellet porosity & 0.35 & \\
$k_{1}$ & reaction rate & $2.418 \times 10^{9}$ & $1 / \mathrm{s}$ \\
$k_{2}$ & reaction rate & $2.706 \times 10^{9}$ & $1 / \mathrm{s}$ \\
$k_{3}$ & reaction rate & $1.013 \times 10^{9}$ & $1 / \mathrm{s}$ \\
$E_{1}$ & exponential factor & $1.129 \times 10^{5}$ & $\mathrm{~J} / \mathrm{mol}$ \\
$E_{2}$ & exponential factor & $1.313 \times 10^{5}$ & $\mathrm{~J} / \mathrm{mol}$ \\
$E_{3}$ & exponential factor & $1.196 \times 10^{5}$ & $\mathrm{~J} / \mathrm{mol}$ \\
$\Delta H_{r 1}$ & heat of reaction & $-1.285 \times 10^{6}$ & $\mathrm{~J} / \mathrm{Kmol}$ \\
$\Delta H_{r 2}$ & heat of reaction & $-3.276 \times 10^{6}$ & $\mathrm{~J} / \mathrm{Kmol}$ \\
$\Delta H_{r 3}$ & heat of reaction & $-4.561 \times 10^{6}$ & $\mathrm{~J} / \mathrm{Kmol}$ \\
$\rho_{f}$ & feed stream density & 0.582 & $\mathrm{~kg} / \mathrm{m}^{3}$ \\
$\rho_{s}$ & catalysts density & 2000 & $\mathrm{~kg} / \mathrm{m}^{3}$ \\
$C_{p f}$ & feed stream heat capacity & 1045 & $\mathrm{~J} / \mathrm{Kg}-\mathrm{K}$ \\
$C_{p c}$ & coolant fluid heat capacity & 483.559 & $\mathrm{~J} / \mathrm{Kg}-\mathrm{K}$ \\
$C_{p s}$ & catalysts heat capacity & 836 & $\mathrm{~J} / \mathrm{Kg}-\mathrm{K}$ \\
$r^{\prime}$ & reactor radius & 0.0125 & $\mathrm{~m}$ \\
$U$ & heat transfer coefficient & 96.02 & $\mathrm{~J} / \mathrm{kg}-\mathrm{K}-\mathrm{s}$ \\
$\mu$ & catalyst activity & 1 & \\
$R$ & ideal gas constant & 8.31447 & $\mathrm{~J} / \mathrm{mol}-\mathrm{K}$ \\
$T^{f}$ & feed stream temperature & 628 & $\mathrm{~K}$ \\
$C_{A}^{f}$ & reactant A feed stream concentration & 0.181 & $\mathrm{kmol} / \mathrm{m}^{3}$ \\
$C_{B}^{f}$ & component B feed stream concentration & 0 & $\mathrm{kmol} / \mathrm{m}^{3}$ \\
$T_{w}$ & heating fluid temperature & 630 & $\mathrm{~K}$ \\
$D_{m}$ & mass diffusivity & $1 \times 10^{-7}$ & $\mathrm{~m}{ }^{2} / \mathrm{s}$ \\
$D_{t}$ & thermal diffusivity & $1 \times 10^{-7}$ & $\mathrm{~m}{ }^{2} / \mathrm{s}$ \\
$M w_{A}$ & compound A molecular weight & 230 & $\mathrm{Kg} / \mathrm{Kgmol}$ \\
$M w_{B}$ & compound B molecular weight & 200 & $\mathrm{Kg} / \mathrm{Kgmol}$ \\
\hline & & &
\end{tabular}

Table 6: Design data for the non-isothermal catalytic fixed-bed tubular reactor case study.

\begin{tabular}{|ccccccccccc|}
\hline Product & $\begin{array}{c}\text { Conversion } \\
\text { Fraction }\end{array}$ & $\begin{array}{c}\text { Demand } \\
\text { rate }[\mathrm{Kg} / \mathrm{s}]\end{array}$ & $\begin{array}{c}\text { Process } \\
\text { cost }[\$ / \mathrm{kg}]\end{array}$ & $\begin{array}{c}\text { Inventory } \\
\text { cost }[\$ / \mathrm{kg}]\end{array}$ & \multicolumn{4}{c}{ Transition Cost $[\$]$} \\
\hline$A$ & 0.5 & 1 & 270 & 1.5 & 0 & 5 & 6 & $D$ & $E$ & $F$ \\
$B$ & 0.6 & 2 & 280 & 1 & 10 & 0 & 5 & 8 & 6 & 10 \\
$C$ & 0.7 & 1.2 & 350 & 2 & 7 & 10 & 0 & 8 & 7 & 11 \\
$D$ & 0.8 & 3 & 380 & 3 & 6 & 10 & 10 & 0 & 12 & 8 \\
$E$ & 0.9 & 1.5 & 430 & 2.5 & 8 & 9 & 10 & 10 & 0 & 10 \\
$F$ & 0.99 & 2 & 380 & 1.2 & 11 & 8 & 7 & 9 & 11 & 0 \\
\hline
\end{tabular}

Table 7: Process data for the non-isothermal catalytic fixed-bed tubular reactor case study. $A, B, C, D, E$ and $F$ stand for the five products to be manufactured. 


\begin{tabular}{|cccccccccccc|}
\hline & & & & & $v$ & $\theta$ & Amount & Production & Process & T start & T end \\
Slot & Prod & $\hat{C}_{A}$ & $\hat{C}_{B}$ & $\hat{T}_{r}$ & {$[\mathrm{~m} / \mathrm{s}]$} & {$[\mathrm{h}]$} & $\begin{array}{c}\text { Produced }[\mathrm{Kg}] \\
\text { rate }[\mathrm{kg} / \mathrm{m}]\end{array}$ & $\begin{array}{c}\text { time }[\mathrm{h}] \\
{[\mathrm{h}]}\end{array}$ \\
\hline 1 & $A$ & 0.5 & 0.442 & 1.007 & 4.879 & 1.6 & 1479.119 & 3745.322 & 0.395 & 0 & 10.39 \\
2 & $B$ & 0.4 & 0.529 & 1.006 & 3.655 & 1.6 & 2958.238 & 3367.079 & 0.879 & 10.39 & 21.27 \\
3 & $E$ & 0.1 & 0.768 & 1.004 & 1.393 & 1.5 & 2218.679 & 1924.178 & 1.153 & 21.27 & 32.42 \\
4 & $F$ & 0.01 & 0.791 & 1.004 & 0.664 & 1.7 & 2958.238 & 1008.935 & 2.932 & 32.42 & 45.36 \\
5 & $D$ & 0.2 & 0.694 & 1.005 & 2.03 & 1.6 & 4437.358 & 2493.723 & 1.779 & 45.36 & 57.14 \\
6 & $C$ & 0.3 & 0.613 & 1.005 & 2.751 & 1.5 & 4174527.473 & 2956.504 & 1411.981 & 57.14 & 1479.12 \\
\hline
\end{tabular}

Table 8: Simultaneous Scheduling and Control results for the non-isothermal catalytic fixed-bed tubular reactor case study. The best production sequence is: $A \rightarrow B \rightarrow E \rightarrow F \rightarrow D \rightarrow C$ featuring $1479.119 \mathrm{~h}$ as total cyclic time and objective function value of $\$ 787919.947$. The optimal solution was computed in $12.08 \mathrm{~h}$ CPU time. $\theta$ stands for transition times. 


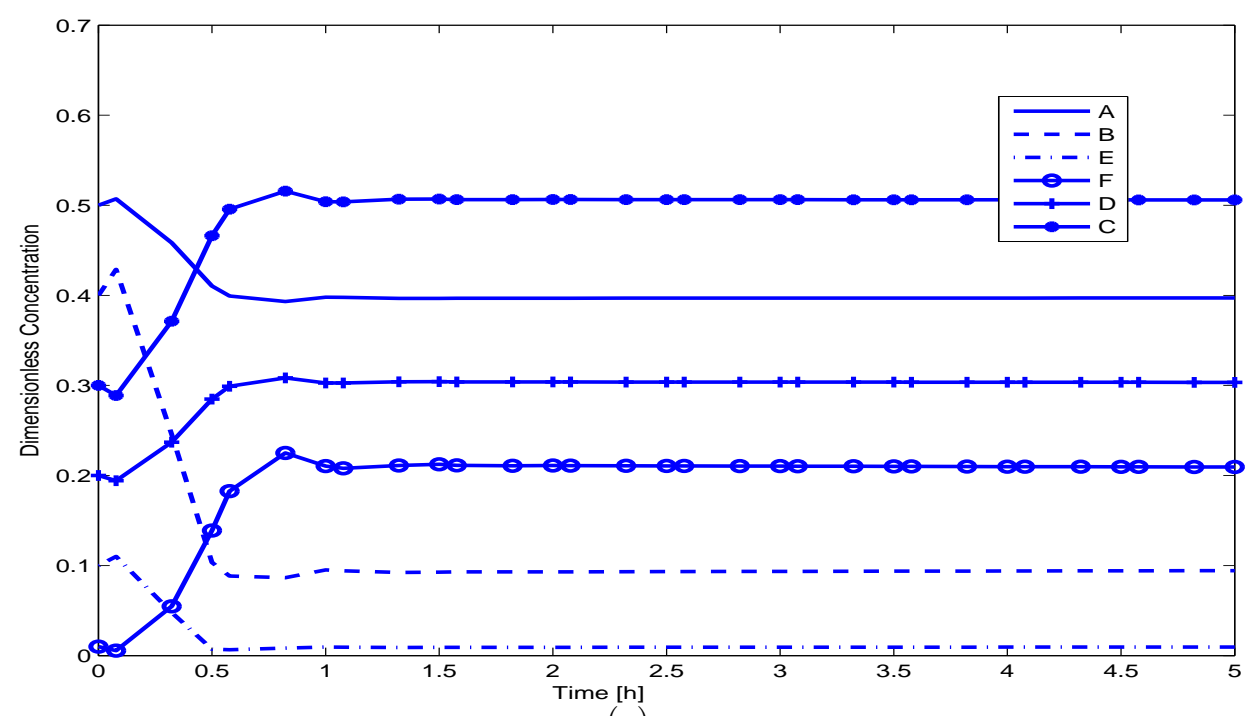

(a)

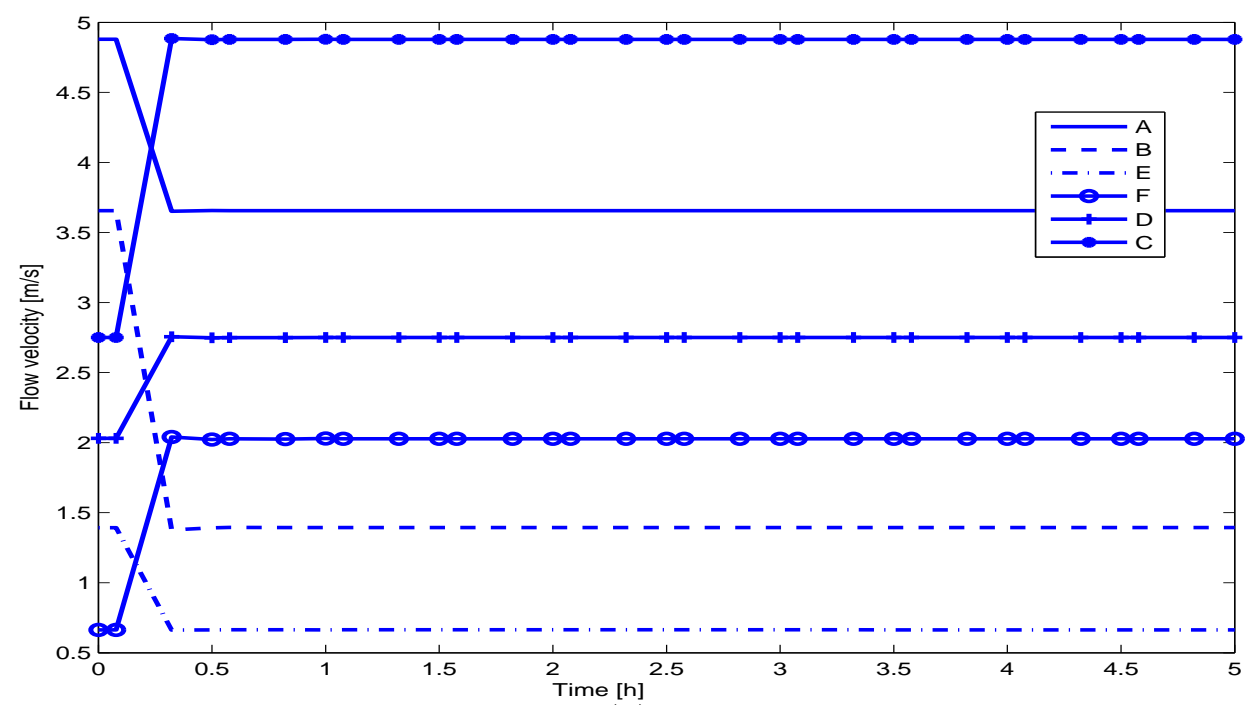

(b)

Figure 5: Optimal dynamic transition profiles for the nonisothermal catalytic fixed-bed tubular reactor. The optimal production sequence is: $A \rightarrow B \rightarrow E \rightarrow F \rightarrow D \rightarrow C$.

Similarly to the past case studies, the optimal dynamic transition responses display a smooth behavior, as shown in Figure 5. Once again, the manipulated variable takes a ramp-like shape, and no inverse system response behavior is observed. This example clearly shows the impact, in terms of CPU time, of increasing both the number of integer variables and the perceived nonlinearity of the distributed parameter model. As a matter fact, in this problem the number of binary variables were 72,22 more integer variables when compared to the second case study. Moreover, the number of continuous variables and constraints between the second and third case studies are similar. However, the computation of the optimal solution 
of the third case study, required around 6 times the CPU to find an optimal solution with the branch and bound method in SBB for the second case study. When realizing that the main difference between these problems lies in the number of binary variables, and that this translates into a larger computational cost, then it is worth to consider alternative MINLP methods (e.g. Generalized Benders Decomposition, Outer-Approximation) or solution approaches such as advanced decomposition techniques [14].

\section{Conclusions}

In this paper we have formulated an extension of our previous work [6], on the simultaneous scheduling and control optimization problem in continuous stirred tank reactors, to incorporate distributed parameter systems. Specifically, we have dealt with nonisothermal one-dimensional plug flow reactors, featuring large dimensionality and highly nonlinear behavior. The proposed simultaneous scheduling and control optimization formulation was able to successfully solve all the addressed problems, demanding different computational requirements. After some trials, we found that because the addressed problems feature simple geometry characteristics, a finite difference scheme [8] was sufficient to discretize the longitudinal component. This will not be necessarily the case for more complex system geometries, and they probably will demand more sophisticated discretization strategies such as finite elements [12] and spectral [13] methods. After spatial discretization, temporal discretization was efficiently handled by using orthogonal collocation on finite elements [9]. Because of the highly nonlinear system behavior, we used the sbb/CONOPT branch and bound procedure [15] to address the solution of the underlying MINLPs.

There are several possible research extensions of the present problem. First, product transitions are more common, but not only, in the polymer industry [3], [17], [18], [19]. Hence, because of complex kinetics, mass and energy interactions and their distributed nature, the scheduling and control problem in polymerization tubular reactors presents a formidable computational challenge. In this context, the computational demands of the problems addressed in this work, suggest that the efficient solution of the MINLPs problems arising from polymerization tubular reactors will demand special decomposition

optimization techniques as the ones we have used for other types of polymerization reactors [20]. Another 
extension of the present work deals with the computation of global optimal solutions. We have made some attempts to compute global optimal solutions of the MINLPs problems addressed in the this work, but without success, even for the first case study that happens to be the simpler problem. The global optimal solution of large scale MINLPs seems to be out of reach for most of the distributed parameter systems, and it is a topic that deservers further research consideration. 


\section{References}

[1] N.K. Read S.X. Zhang and W.H. Ray. Runaway Phenomena in Low-Density Polyethylene Autoclave Reactors. AIChE J., 42(10):2911-2925, 1996.

[2] T. Bhatia and L.T. Biegler. Dynamic Optimization in the Design and Scheduling of Multiproduct Batch Plants. Ind. Eng. Chem. Res., 35:2234-2246, 1996.

[3] A.C. Allcock R. Mahadevan, F.J. Doyle III. Scheduling of Polymer Grade Transitions. AIChE J., 48(8):1754-1764, 2002.

[4] B. V. Mishra, E. Mayer, J. Raisch, and A. Kienle. Short-Term Scheduling of Batch Processes. A Comparative Study of Differentes Approaches. Ind. Eng. Chem. Res., 44:4022-4034, 2005.

[5] R. H. Nystrom, R. Franke, I. Harjunkoski, and A. Kroll. Production Campaing Planning Including Grade Transition Sequencing and Dynamic Optimization. Comput. Chem. Eng., 29(10):2163-2179, 2005.

[6] A. Flores-Tlacuahuac and I.E. Grossmann. Simultaneous Cyclic Scheduling and Control of a Multiproduct CSTR. Ind. Eng. Chem. Res., 45(20):6175-6189, 2006.

[7] S. Terrazas-Moreno, A. Flores-Tlacuauhuac, and I.E. Grossmann. Simultaneous scheduling and control in polymerization reactors. AIChE J., 53(9), 2007.

[8] W. E. Schiesser. The Numerical Method of Lines. Integration of Partial Differential Equations. Academic Press, Inc., 1991.

[9] L.T. Biegler. An overview of simultaneous strategies for dynamic optimization. Chemical Engineering and Processing, 46(11):1043-1053, 2007.

[10] Pierre Bonami, Lorenz Biegler, Andrew Conn, Gerard Cornuejols, Ignacio Grossmann, Carl Laird, Jon Lee, Andrea Lodi, Francois Margot, Sawaya Nicolas, and Andreas Waechter. An algorithmic framework for convex mixed integer nonlinear programs. Discrete Optimization, doi:10.1016/j.disopt.2006.10.011, 2007. 
[11] J.M. Pinto and I. E. Grossmann. Optimal Cyclic Scheduling of Multistage Continuous Multiproduct Plants. Comput. Chem. Eng., 18(9):797-816, 1994.

[12] Prodromos Daoutidis Nikolaos V. Mantzaris and Friedrich Srienc. Numerical solution of multi-variable cell population balance models: III. Finite element methods. Comput. Chem. Eng., 25:1463-1481, 2001.

[13] Prodromos Daoutidis Nikolaos V. Mantzaris and Friedrich Srienc. Numerical solution of multi-variable cell population balance models: II. Spectral methods. Comput. Chem. Eng., 25:1441-1462, 2001.

[14] M. Guinard and S.Kim. Lagrangean Decomposition: A model yielding Stronger Lagrangean Bounds. Mathematical Programming, 39:215-228, 1987.

[15] A. Brooke, D. Kendrick, Meeraus, and R. A. Raman. GAMS: A User's Guide. GAMS Development Corporation, 1998, http://www.gams.com.

[16] W. Wu and S.Y. Ding. Model Predictive Control of Nonlinear Distributed Parameter Systems Using Spatial Neural-Networks Architectures. Ind. Eng. Chem. Res., 47:7264-7273, 2008.

[17] J.R. Richards J.P. Congalidis and W.H. Ray. Scheduling of Polymer Grade Transitions. AIChE J., 48(8):1754-1764, 2002.

[18] C. Chatzidoukas, C. Kiparissidis, J.D. Perkins, and Pistikopoulos E.N. Optimal Grade Transition Campaign Scheduling in a Gas Phase Polyolefin FBR Using Mixed Integer Dynamic Optimization. Process System Engineering, pages 744-747. Elsevier, 2003.

[19] A. Kroll W. Marquardt A. Prata, J.Oldenburg. Integrated Scheduling and Dynamic Optimization of Grade Transitions for a Continuous Polymerization Reactor. Comput. Chem. Eng., 32:463-476, 2008.

[20] Sebastian Terrazas-Moreno, Antonio Flores-Tlacuahuac, and Ignacio E. Grossmann. A Lagrangean Heuristic Approach for the Simultaneous Cyclic Scheduling and Optimal Control of Multi-Grade Polymerization Reactors. AIChE J., 54(1):163-182, 2008. 


\section{A Simultaneous Scheduling and Control Optimization Formu-}

\section{lation}

This section contains the simultaneous scheduling and control optimization formulation previously published in [6] and it has been included in the present work only for completeness reasons. A complete description of the meaning of the objective function and constraints can be found elsewhere [6].

All the indices, decision variables and system parameters used in the SSC MIDO problem formulation are as follows:

1. Indices

Products

Slots

Finite elements

Collocation points

System states

Manipulated variables

$$
i, p=1, \ldots N_{p}
$$

$k=1, \ldots N_{s}$

$$
f=1, \ldots N_{f e}
$$

$c, l=1, \ldots N_{c p}$

$n=1, \ldots N_{x}$

$m=1, \ldots N_{u}$ 
2. Decision variables

$y_{i k} \quad$ Binary variable to denote if product $i$ is assigned to slot $k$

$y_{i k}^{\prime} \quad$ Binary auxiliary variable

$z_{i p k} \quad$ Binary variable to denote if product $i$ is followed by product $p$ in slot $k$

$p_{k} \quad$ Processing time at slot $k$

$t_{k}^{e} \quad$ Final time at slot $k$

$t_{k}^{s} \quad$ Start time at slot $k$

$G_{i} \quad$ Production rate

$T_{c} \quad$ Total production wheel time $[\mathrm{h}]$

$x_{f c k}^{n} \quad$ N-th system state in finite element $f$ and collocation point $c$ of slot $k$

$u_{f c k}^{m} \quad$ M-th manipulated variable in finite element $f$ and collocation point $c$ of slot $k$

$W_{i} \quad$ Amount produced of each product $[\mathrm{kg}]$

$\theta_{i k} \quad$ Processing time of product $i$ in slot $k$

$\theta_{k}^{t} \quad$ Transition time at slot $k$

$\Theta_{i} \quad$ Total processing time of product $i$

$x_{o, f k}^{n} \quad n$-th state value at the beginning of the finite element $f$ of slot $k$

$\bar{x}_{k}^{n} \quad$ Desired value of the $n$-th state at the end of slot $k$

$\bar{u}_{k}^{m} \quad$ Desired value of the $m$-th manipulated variable at the end of slot $k$

$x_{i n, k}^{n} \quad n$-th state value at the beginning of slot $k$

$u_{i n, k}^{n} \quad m$-th manipulated variable value at the beginning of slot $k$

$X_{i} \quad$ Conversion

\section{Parameters}

$N_{p} \quad$ Number of products

$N_{s} \quad$ Number of slots

$N_{f e} \quad$ Number of finite elements

$N_{c p} \quad$ Number of collocation points

$N_{x} \quad$ Number of system states

$N_{u} \quad$ Number of manipulated variables 
$D_{i} \quad$ Demand rate $[\mathrm{kg} / \mathrm{h}]$

$C_{i}^{p} \quad$ Price of products $[\$ / \mathrm{kg}]$

$C_{i}^{s} \quad$ Cost of inventory

$C^{r} \quad$ Cost of raw material

$h_{f k} \quad$ Length of finite element $f$ in slot $k$

$\Omega_{c c} \quad$ Matrix of Radau quadrature weights

$\bar{x}_{k}^{n} \quad$ Desired value of the $n$-th system state at slot $k$

$\bar{u}_{k}^{m} \quad$ Desired value of the $m$-th manipulated variable at slot $k$

$\theta^{\max } \quad$ Upper bound on processing time

$t_{i p}^{t} \quad$ Estimated value of the transition time between product $i$ and $p$

$x_{s s, i}^{n} \quad n$-th state steady value of product $i$

$u_{s s, i}^{m} \quad m$-th manipulated variable value of product $i$

$F^{o} \quad$ Feed stream volumetric flow rate

$X_{i} \quad$ Conversion degree

$x_{\min }^{n}, x_{\max }^{n}$ Minimum and maximum value of the state $x^{n}$

$u_{\min }^{m}, u_{\max }^{m}$ Minimum and maximum value of the manipulated variable $u^{m}$

$\gamma_{c} \quad$ Roots of the Lagrange orthogonal polynomial

\section{- Objective function.}

$$
\begin{aligned}
\max & \left\{\sum_{i=1}^{N_{p}} \frac{C_{i}^{p} W_{i}}{T_{c}}-\sum_{i=1}^{N_{p}} \frac{C_{i}^{s}\left(G_{i}-W_{i}\right)}{2 \Theta_{i} T_{c}}-\sum_{k=1}^{N_{s}} \sum_{f=1}^{N_{f e}} h_{f k} \sum_{c=1}^{N_{c p}} \frac{C^{r} t_{f c k} \Omega_{c, N_{c p}}}{T_{c}}\left(\left(x_{f c k}^{1}-\bar{x}_{k}^{1}\right)^{2}\right.\right. \\
& \left.\left.+\ldots+\left(x_{f c k}^{n}-\bar{x}_{k}^{n}\right)^{2}+\left(u_{f c k}^{1}-\bar{u}_{k}^{1}\right)^{2}+\ldots+\left(u_{f c k}^{m}-\bar{u}_{k}^{m}\right)^{2}\right)\right\}
\end{aligned}
$$

1. Scheduling part. 
a) Product assignment

$$
\begin{aligned}
\sum_{k=1}^{N_{s}} y_{i k} & =1, \forall i \\
\sum_{i=1}^{N_{p}} y_{i k} & =1, \forall k \\
y_{i k}^{\prime} & =y_{i, k-1}, \forall i, k \neq 1 \\
y_{i, 1}^{\prime} & =y_{i, N_{s}}, \forall i
\end{aligned}
$$

b) Amounts manufactured

$$
\begin{aligned}
& W_{i} \geqslant D_{i} T_{c}, \forall i \\
& W_{i}=G_{i} \Theta_{i}, \forall i \\
& G_{i}=F^{o}\left(1-X_{i}\right), \forall i
\end{aligned}
$$

c) Processing times

$$
\begin{aligned}
\theta_{i k} & \leqslant \theta^{\max } y_{i k}, \forall i, k \\
\Theta_{i} & =\sum_{k=1}^{N_{s}} \theta_{i k}, \forall i \\
p_{k} & =\sum_{i=1}^{N_{p}} \theta_{i k}, \forall k
\end{aligned}
$$

d) Transitions between products

$$
z_{i p k} \geqslant y_{p k}^{\prime}+y_{i k}-1, \forall i, p, k
$$


e) Timing relations

$$
\begin{aligned}
\theta_{k}^{t} & =\sum_{i=1}^{N_{p}} \sum_{p=1}^{N_{p}} t_{p i}^{t} z_{i p k}, \forall k \\
t_{1}^{s} & =0 \\
t_{k}^{e} & =t_{k}^{s}+p_{k}+\sum_{i=1}^{N_{p}} \sum_{p=1}^{N_{p}} t_{p i}^{t} z_{i p k}, \forall k \\
t_{k}^{s} & =t_{k-1}^{e}, \forall k \neq 1 \\
t_{k}^{e} & \leqslant T_{c}, \forall k \\
t_{f c k} & =(f-1) \frac{\theta_{k}^{t}}{N_{f e}}+\frac{\theta_{k}^{t}}{N_{f e}} \gamma_{c}, \forall f, c, k
\end{aligned}
$$

2. Dynamic Optimization part.

a) Dynamic mathematical model discretization

$$
x_{f c k}^{n}=x_{o, f k}^{n}+\theta_{k}^{t} h_{f k} \sum_{l=1}^{N_{c p}} \Omega_{l c} \dot{x}_{f l k}^{n}, \forall n, f, c, k
$$

b) Continuity constraint between finite elements

$$
x_{o, f k}^{n}=x_{o, f-1, k}^{n}+\theta_{k}^{t} h_{f-1, k} \sum_{l=1}^{N_{c p}} \Omega_{l, N_{c p}} \dot{x}_{f-1, l, k}^{n}, \forall n, f \geqslant 2, k
$$

c) Model behavior at each collocation point

$$
\dot{x}_{f c k}^{n}=f^{n}\left(x_{f c k}^{1}, \ldots, x_{f c k}^{n}, u_{f c k}^{1}, \ldots u_{f c k}^{m}\right), \forall n, f, c, k
$$


d) Initial and final controlled and manipulated variable values at each slot

$$
\begin{aligned}
x_{i n, k}^{n} & =\sum_{i=1}^{N_{p}} x_{s s, i}^{n} y_{i, k}, \forall n, k \\
\bar{x}_{k}^{n} & =\sum_{i=1}^{N_{p}} x_{s s, i}^{n} y_{i, k+1}, \forall n, k \neq N_{s} \\
\bar{x}_{k}^{n} & =\sum_{i=1}^{N_{p}} x_{s s, i}^{n} y_{i, 1}, \forall n, k=N_{s} \\
u_{i n, k}^{m} & =\sum_{i=1}^{N_{p}} u_{s s, i}^{m} y_{i, k}, \forall m, k \\
\bar{u}_{k}^{m} & =\sum_{i=1}^{N_{p}} u_{s s, i}^{m} y_{i, k+1}, \forall m, k \neq N_{s}-1 \\
\bar{u}_{k}^{m} & =\sum_{i=1}^{N_{p}} u_{s s, i}^{m} y_{i, 1}, \forall m, k=N_{s} \\
x_{N_{f e}, N_{c p}, k}^{n} & =\bar{x}_{k}^{n}, \forall n, k \\
u_{1,1, k}^{m} & =u_{i n, k}^{m}, \forall m, k \\
u_{N_{f e}, N_{c p}, k}^{m} & =\bar{u}_{i n, k}^{m}, \forall m, k \\
x_{o, 1, k}^{n} & =x_{i n, k}^{n}, \forall n, k
\end{aligned}
$$

e) Lower and upper bounds on the decision variables

$$
\begin{aligned}
& x_{\text {min }}^{n} \leqslant x_{f c k}^{n} \leqslant x_{\text {max }}^{n}, \forall n, f, c, k \\
& u_{\text {min }}^{m} \leqslant u_{f c k}^{m} \leqslant u_{\text {max }}^{m}, \forall m, f, c, k
\end{aligned}
$$

